Gate tunable layer selectivity of transport in bilayer graphene nanostructures

This content has been downloaded from IOPscience. Please scroll down to see the full text. 2016 EPL 11317006

(http://iopscience.iop.org/0295-5075/113/1/17006)

View the table of contents for this issue, or go to the journal homepage for more

Download details:

IP Address: 146.175.11.185

This content was downloaded on 21/03/2016 at 10:33

Please note that terms and conditions apply. 


\title{
Gate tunable layer selectivity of transport in bilayer graphene nanostructures
}

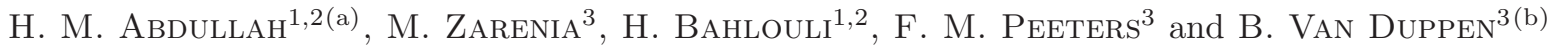 \\ 1 Department of Physics, King Fahd University of Petroleum and Minerals - 31261 Dhahran, Saudi Arabia \\ 2 Saudi Center for Theoretical Physics - 31261 Dhahran, Saudi Arabia \\ 3 Department of Physics, University of Antwerp - Groenenborgerlaan 171, B-2020 Antwerp, Belgium
}

received 6 November 2015; accepted in final form 13 January 2016

published online 28 January 2016

PACS 72.80.Vp - Electronic transport in graphene

PACS 73.21.Ac - Multilayers

PACS 73.22.Pr - Electronic structure of graphene

\begin{abstract}
Recently it was found that bilayer graphene may exhibit regions with and without van der Waals coupling between the two layers. We show that such structures can exhibit a strong layer selectivity when current flows through the coupled region and that this selectivity can be tuned by means of electrostatic gating. Analysing how this effect depends on the type of bilayer stacking, the potential on the gates and the smoothness of the boundary between the coupled and decoupled regions, we show that nearly perfect layer selectivity is achievable in these systems. This effect can be further used to realise a tunable layer switch.
\end{abstract}

Copyright (c) EPLA, 2016

Research aiming at the usage of graphene as a basis for the next generation of electronics has been very intense during the last few years [1-5]. Many proposals using graphene sheets have been made based on varying carrier mobility [6] or using the energy valleys as a basis for valleytronics [7]. If one changes the size of the flake into graphene quantum dots and nanoribbons, energy gaps can be controlled through size quantization [8-10].

To generate band gaps in the system, researchers have considered graphene composite structures such as bilayer graphene $[11,12]$, a set of two graphene layers coupled by weak van der Waals interaction. It has been shown that, depending on the stacking configuration, one can open a band gap in this system by electrostatic gating [13-15]. Furthermore, different nanostructures where the bilayer was deformed also received a lot of attention. One interesting example is a twisted bilayer structure where the two graphene layers are rotated with respect to each other. The electronic structure of such systems feature very peculiar properties [16-19].

In this work we investigate the transport properties of another graphene composite made of two layers. Now the layers are only locally coupled by van der Waals interaction into a bilayer structure (see fig. 1(a)). By locally

\footnotetext{
(a) E-mail: alshehab211@gmail.com

(b) E-mail: ben . vanduppen@uantwerpen . be
}

gating the coupled region we show that it is possible to select the layer in which the current flows after having tunnelled through the joined region. In this way the structure constitutes a gate tunable layer switch.

Recently such locally coupled structures have been observed in chemical vapor deposition (CVD) graphene samples [20-22] where, due to rippling, the layers were decoupled in some regions while being connected in others. The proposed structure can be subsequently created by conventional etching techniques traditionally used to, e.g., create graphene nanoribbons [23,24].

We model the layer switch as two single layers of graphene (SLG) that are locally joined together by van der Waals forces into a bilayer graphene (BLG) structure as depicted in fig. 1(a). There are two different ways the layers can be joined together depending on the relative stacking: 1) exactly aligning both honeycomb lattices on top of each other, yielding an AA-stacked configuration, or 2) relatively shifting one layer by one inter-atomic distance such that only two sublattices are aligned, corresponding to an AB-stacked configuration. We found that the type of stacking configuration strongly influences the layer selectivity of the system.

We perform our calculation for abrupt and smooth SLGBLG interfaces (see fig. 1(b), (c). These types of interfaces show no quantitative or qualitative differences indicating that the switching effect is robust against the smoothing of 


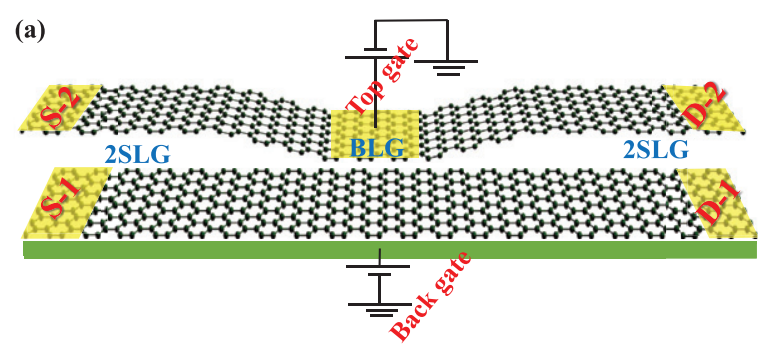

(b)
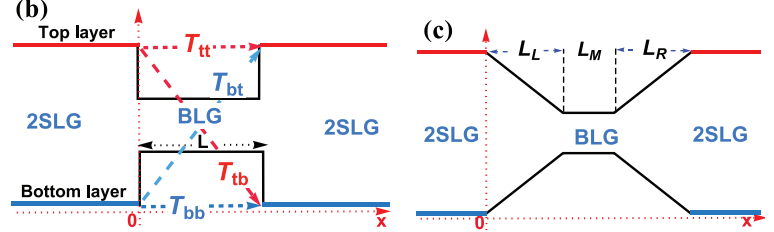

Fig. 1: (Colour online) (a) Schematic presentation of the proposed structure with the indication of the top and back gate, the coupled region (BLG) and the decoupled graphene sheets (2SLG). (b) and (c): cross-section of the device with abrupt and smooth transitions from the coupled to the decoupled region, respectively. The arrows in (b) indicate the different transmission channels as discussed in the text.

the SLG-BLG junction and that we can restrict ourselves to the model of abrupt interfaces.

We describe the dynamics of the carriers in the different regions by the continuum Hamiltonian written in the basis of atomic orbitals $\boldsymbol{\Psi}=\left(\boldsymbol{\Psi}_{1}, \boldsymbol{\Psi}_{2}\right)^{T}$ with $\boldsymbol{\Psi}_{i}=\left(\Psi_{i A}, \Psi_{i B}\right)$ being the SLG spinor. Then the Hamiltonian of the system reads $[2,25,26]$

$$
H=\left[\begin{array}{cc}
H_{1} & C_{\tau, \zeta} \\
C_{\tau, \zeta}^{\dagger} & H_{2}
\end{array}\right],
$$

where $H_{i}=v_{F} \vec{\sigma} \cdot \vec{p}+V_{i}$ is the SLG Hamiltonian with $v_{F}=$ $10^{6} \mathrm{~m} / \mathrm{s}$ the carrier Fermi velocity and $V_{i}$ the electrostatic potential on the $i$-th layer which can be varied by gating the sample with top and back gates as shown in fig. 1(a). In eq. (1) $C_{\tau, \zeta}$ is the inter-layer coupling and is defined by

$$
C_{\tau, \zeta}=\left[\begin{array}{cc}
\tau \gamma_{1} & 0 \\
\zeta \gamma_{1} & \tau \gamma_{1}
\end{array}\right],
$$

where $\gamma_{1}$ is the dominant inter-layer hopping parameter which has the value $\gamma_{1} \approx 0.4 \mathrm{eV}$ for AB-stacking [27] and $\gamma_{1} \approx 0.2 \mathrm{eV}$ for AA-stacking [28-30]. The coupling between the two graphene layers is controlled by the parameters $\tau$ and $\zeta$ that can "switch on" or "switch off" the inter-layer coupling between specific sublattices and therefore we can model different stacking types by assigning different values to these parameters. For $\tau=\zeta=0$, the two layers are decoupled and the Hamiltonian represents two independent SLG sheets. To achieve AA-stacking we select $\tau=1$ and $\zeta=0$ while for AB-stacking we need $\tau=0$ and $\zeta=1$. The energy spectra of the Hamiltonian from eq. (1) are shown in fig. 2. Note that in eq. (2) we neglected the skew inter-layer hopping parameters corresponding to the sublattices which are not directly above

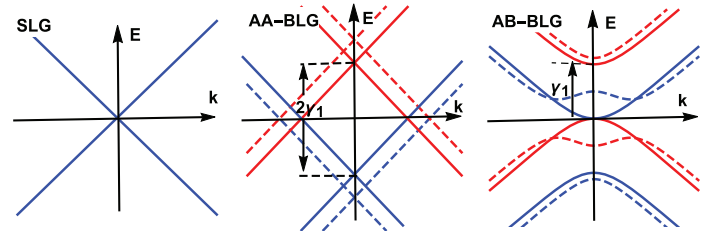

Fig. 2: (Colour online) Band dispersion relations around the Dirac point for single-layer graphene (left), AA-stacked (middle) and AB-stacked (right) bilayer graphene. The dashed curves correspond to the spectrum of the system in the case of a finite bias.

each other. The final result does not depend strongly on this assumption since these hopping parameters are off diagonal contributions accompanied by a term linear in the momentum [25].

To determine the layer selectivity we first note that translation symmetry in the $y$-direction implies conservation of $k_{y}$. Then we connect the eigenstates of each region defined in fig. 1(b) as "2SLG" for the decoupled single layer and "BLG" for the bilayer regions at the intermediate boundaries by matching each sublattice spinor component with its counterpart in the other region.

The eigenstates in the 2 SLG regions consist of a set of oppositely propagating states in each layer separately. This allows us to define a layer resolved current by applying appropriate boundary conditions and identifying the coefficients of each mode with electrons propagating in one of the two SLG layers. In this manner we can calculate the inter- and intra-layer transmission and reflection probabilities as the square of the modulus [31-33]. The transmission probabilities are denoted by $T_{t t}$ or $T_{b b}$ for intra-layer transitions and by $T_{t b}$ or $T_{b t}$ for inter-layer transitions. The different transmission channels that are considered in this work are illustrated in fig. 1(b). The reflection probabilities are denoted as $R_{t t}$ and $R_{b b}$ or $R_{t b}$ and $R_{b t}$ following the same convention. To conserve probability these quantities are normalized such that $T_{i i}+T_{i j}+R_{i i}+R_{i j}=1$, $(i, j)=t, b$.

The number of transmission channels depends on the availability of propagating modes in the BLG part of the device [25]. As can be inferred from the energy spectra depicted in fig. 2, for each energy it is possible to have up to two modes of propagation (indicated in red and blue in fig. 2). Note that for the AA-stacked BLG part there are always two propagating modes, while for AB-BLG it is possible to have none if the energy is in the gap, one if the energy is below the second band (i.e. $E<\gamma_{1}$ ) or two if the energy is sufficiently high.

In order to take into account a smooth transition between the 2SLG and BLG regions, we used the transfer matrix approach $[31,32,34]$. This approach subdivides the smooth transition into a series of steps. We assume that the inter-layer hopping $\gamma_{1}$ increases linearly from zero in the SLG region until $\gamma_{1}$ in the joined region as shown in fig. 1(c). 
We derive the conductance of the sample from the transmission coefficients using the Landauer-Büttiker [35] formula defined by

$$
G_{i j}^{T}=G_{0} L_{y} \sum_{k_{y}} T_{i j}\left(k_{y}\right),
$$

where $G_{0}=4 e^{2} / h$ and $L_{y}$ is the length of the sample in the $y$-direction. Analogously we can also obtain the reflectance as

$$
G_{i j}^{R}=G_{0} L_{y} \sum_{k_{y}} R_{i j}\left(k_{y}\right),
$$

which gives a measure for the amount of current that is reflected at the junction back into the leads where it came from or into the other layer. These quantities can be used to define a measure of layer filtering as follows:

$$
F_{i j}^{A}=\frac{G_{i j}^{A}}{G_{i, \text { tot }}^{A}},
$$

where $G_{i, \text { tot }}^{A}=G_{i t}^{A}+G_{i b}^{A}$ and $A$ stands either for $T$ or $R$ such that, for example, $F_{b t}^{T}$ measures the filtering to the top layer $(t)$ in the transmission channel $(T)$ when the particle was incident in the bottom layer $(b)$.

In fig. 3 we show the transmission and reflection probabilities as a function of the Fermi energy at normal incidence for different bias $\delta=\left(V_{1}-V_{2}\right) / 2$, and potential strength $v_{0}=\left(V_{1}+V_{2}\right) / 2$ for an AB-stacked junction region. In figs. $3(\mathrm{a})-(\mathrm{d})$ the structure is ungated $\left(v_{0}=0\right)$ and unbiased $(\delta=0)$ with solid (dashed) lines for abrupt (smoothed) interface. $T_{b b}$ and $T_{t b}$ are equal for energies less than the inter-layer hopping $\gamma_{1}$. In contrast, for $E \gtrsim \gamma_{1}$, the electrons strongly scatter from the top (bottom) layer on the left lead into the bottom (top) layer on the right. This difference is due to the presence of two possible transmission channels in BLG while only one channel exists for $E<\gamma_{1}$. On the other hand, the reflection probability shows no layer selectivity except for $E=0$ where electrons completely reflect into the same layer. This is because at this energy there are no available propagating states in BLG.

Applying an electric field on BLG $\left(v_{0}>0\right)$ will shift the energy spectrum and affect the transmission probability as shown in figs. 3(e), (f). The high selectivity or strong scattering between the layers is preserved in the region where the two channels are available, i.e. in the energy intervals $0<E<v_{0}-\gamma_{1}$ and $E>v_{0}+\gamma_{1}$. Similarly, the reflection probability here has no significant selectivity and the strong reflection in $R_{b b}$ channel is shifted by $v_{0}$ since it occurs now at $E=v_{0}$.

In the case when BLG is biased $(\delta>0)$ and gated $\left(v_{0}>0\right)$, the band gap induced by the inter-layer bias suppresses the transmission in the energy region between $v_{0} \pm \delta$, as seen in figs. $3(\mathrm{~g}),(\mathrm{h})$. Moreover, $T_{b b}\left(R_{b b}\right)$ and $T_{t t}\left(R_{t t}\right)$ are almost the same, except in the region where there is only one channel in the BLG region. However,
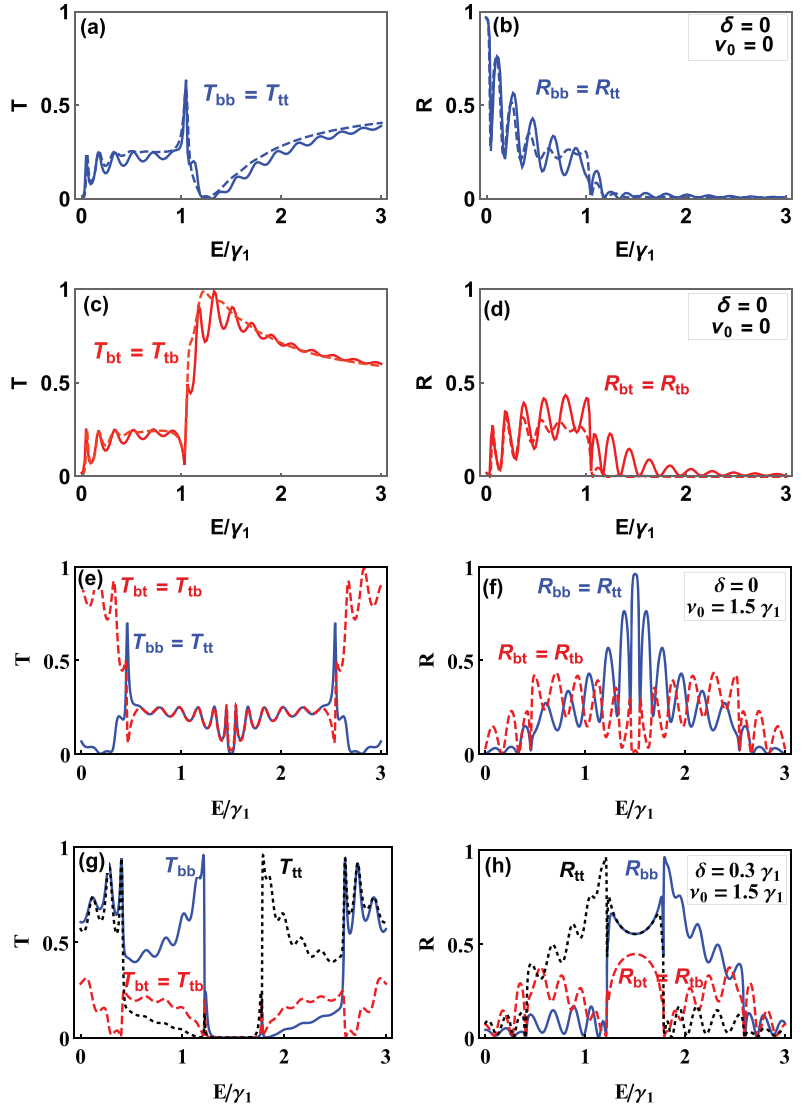

Fig. 3: (Colour online) Transmission and reflection probabilities at normal incidence as a function of the Fermi energy, through 2SLG-AB-2SLG structure. (a), (b) and (c), (d) are for the non-scattered and scattered channels with $\delta=v_{0}=0$, respectively, solid (dashed) lines for abrupt (smoothed) structure with $L=25 \mathrm{~nm}, L_{L}=L_{R}=5 \mathrm{~nm}$ and $L_{M}=20 \mathrm{~nm}$, (see fig. 1(c)). For only abrupt structure with $v_{0}=3 \gamma_{1} / 2$, $((\mathrm{e}),(\mathrm{f}))$ and $((\mathrm{g}),(\mathrm{h}))$ for $\delta=0,0.3 \gamma_{1}$, respectively.

$T_{b t}=T_{t b}$ for all energies, which is a manifestation of a breaking of the inter-layer sublattice equivalence [25].

Due to the finite size of the interaction region, the propagating mode in the BLG region interferes with itself resulting in oscillations of the transmission probabilities, see figs. 3(a), (c). These so-called Fabry-Pérot resonances appear at energies quantized as [26]

$$
E_{B L G}^{n}=\frac{1}{2}\left(-1 \pm \sqrt{1+4 l^{2}\left(\frac{n \pi}{L}\right)^{2}}\right),
$$

where $l=\frac{\hbar v_{F}}{\gamma_{1}} \approx 1.7 \mathrm{~nm}$ (AB-stacking) and $l \approx 3.3 \mathrm{~nm}$ (AA-stacking) is the inter-layer coupling length.

The effect of smoothing the SLG-BLG interface on the transmission and reflection probabilities is shown in fig. 3. In this figure we show the non-scattered and scattered channels in fig. 3(a), (b) and (c), (d), respectively, for abrupt (solid curves) and smoothed (dashed curves) structures. Our results show that the only effect of the smoothness is to remove the oscillations in the transmission 

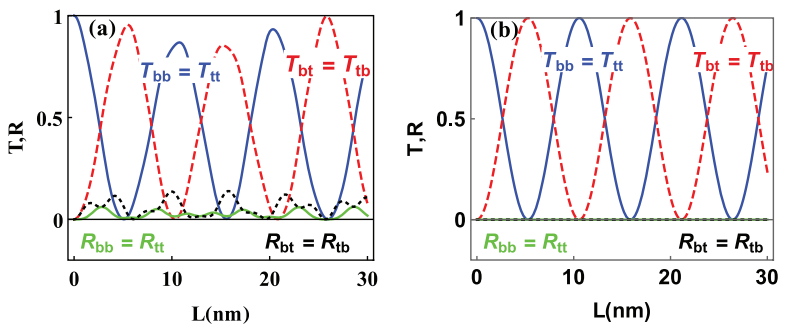

Fig. 4: (Colour online) Transmission and reflection probabilities at normal incidence as a function of the bilayer width $L$ for $\delta=v_{0}=0$. (a) for AB-stacking with $E=3 \gamma_{1} / 2$ and (b) for AA-stacking with $E=3 \gamma_{1} / 2$.

and reflection probabilities. However, the qualitative and quantitative behaviour of the transmission and reflection coefficients is preserved and the selectivity is not significantly influenced by the smoothness.

Figure 4 shows the AB-BLG (left panel) and AA-BLG (right panel) width dependence of the transmission and reflection probabilities for normal incidence. For both types of stacking, $T_{t t}$ and $T_{b b}$ are equal and oscillate with the width of the BLG, while the transmission $T_{b t}=T_{t b}$ oscillates out of phase. This behaviour is due to the interference of the two propagating modes in BLG, but for AB-BLG this will not hold for $E \lesssim \gamma_{1}$, because there is only one propagating mode in this case. On the other hand, the reflection probabilities for AA-stacking are zero for the whole range of $L$, which is due to Klein tunnelling, while for AB-stacking we still have partial reflection. The location of the resonances in $T_{b b}\left(T_{b t}\right)$ for $\mathrm{AA}$ and $\mathrm{AB}$ stacking is given by [9]

$$
L_{m}=\xi \pi l(m+\eta)
$$

with $m$ an integer, $\xi=1$ for AA-stacking and $\xi=2$ for AB-stacking and $\eta=0, \frac{1}{2}$ for $T_{b b}$ and $T_{b t}$, respectively. The different value for $\xi$ is due to the fact that when the crystal is AB-stacked only half of the atoms contribute in the inter-layer coupling while all atoms participate in AA-stacking.

The layer filtering can be controlled by applying an electric field which shifts the potential of the total structure by an amount $v_{0}>0$ and applies a finite potential difference between the layers $\delta>0$. In fig. 5 we present the conductance and reflectance with the corresponding layer filtering as a function of bias for AB-BLG. The conductance shows a certain complementarity: the maxima in the $G_{b b}^{T}\left(G_{t t}^{T}\right)$ coincide with the minima in $G_{b t}^{T}\left(G_{t b}^{T}\right)$ as seen in fig. 5(a). This behaviour clearly shows up in the layer filtering as shown in fig. $5(\mathrm{c})$, where for $\delta \approx-0.35 \gamma_{1}$ the particle is incident on the bottom layer and then is transmitted into the same layer, resulting in $F_{b b}^{T} \approx 1$. While for $\delta=0$ we find that $F_{b t}^{T}=1$, which means that the particle is incident on the bottom layer and is then transmitted into the top layer on the other side of the junction. This result demonstrates that the structure under consideration has a gate tunable layer selectivity and that it can
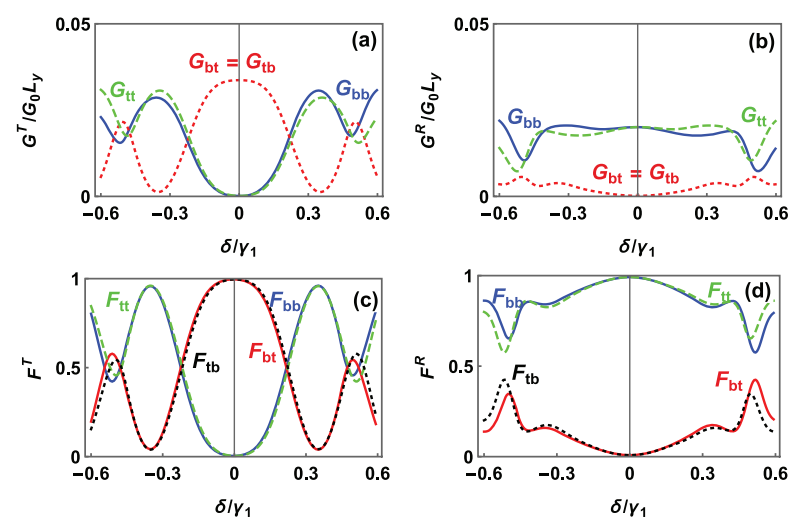

Fig. 5: (Colour online) Conductance and reflectance along with their associated layer filtering as a function of the bias through a 2SLG-AB-2SLG structure for $E=0.3 \gamma_{1}$ and $v_{0}=1.8 \gamma_{1}$ and $L=25.8 \mathrm{~nm}$. (a) and (b): conductance and reflectance; (c) and (d): the corresponding layer filtering, respectively.
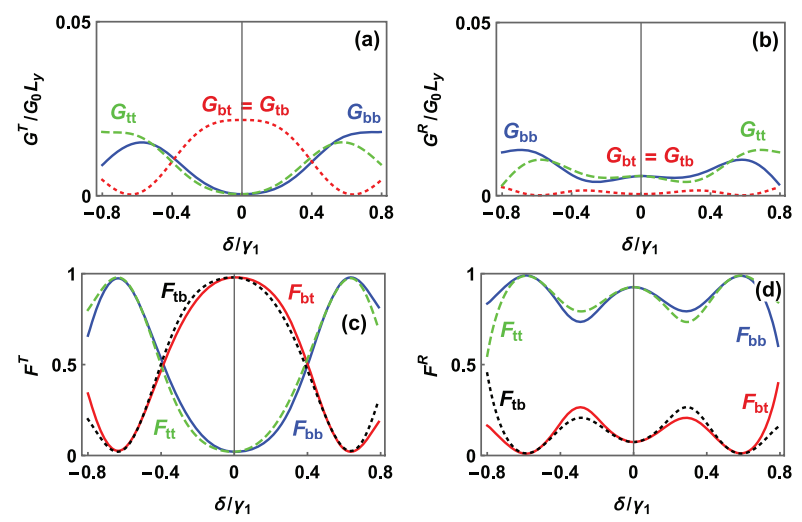

Fig. 6: (Colour online) Conductance and reflectance along with their associated layer filtering as a function of the bias through 2SLG-AA-2SLG structure for $E=0.3 \gamma_{1}$ and $v_{0}=1.8 \gamma_{1}$ and $L=26.4 \mathrm{~nm}$. (a) and (b): conductance and reflectance; (c) and (d): the corresponding layer filtering, respectively.

act as a layer switch. Indeed, by tuning the voltage across the joined region, one can switch the transmitted current between the two layers.

The reflectance with the corresponding layer filtering is shown in figs. $5(\mathrm{~b}),(\mathrm{d})$, respectively. We see small oscillations in $G_{b b}^{R}\left(G_{t t}^{R}\right)$ which are out of phase compared to $G_{b t}^{R}\left(G_{t b}^{R}\right)$ with weak current flow. The results show that also the reflection can be tuned by changing the bias potential $\delta$, but not with the strong selectivity that is possible in the transmission channel.

Also for the AA-stacked variant the scattered and nonscattered conductances are exactly out of phase as shown in fig. 6(a). The layer filtering is nearly perfect for $\delta \approx$ $\pm 0.63 \gamma_{1}$ with $F_{b b}^{T} \approx 1$ and $F_{b t}^{T} \approx 0$, whereas at $\delta \approx 0$ the particles are completely scattered between the layers with $F_{b t}^{T} \approx 1$ and $F_{b b}^{T} \approx 0$ as shown in fig. 6(c). The same analogy here applies to the reflectance and the associated layer filtering as shown in figs. 6(b), (d). 
In summary, we have investigated a system consisting of two locally coupled graphene sheets and have shown that it is a promising candidate for application as a layer switch. We show that independently of the BLG stacking configuration, the layer selectivity can be controlled by an applied gate potential. The different peculiarities associated to the two stacking configurations for achieving maximal switching behaviour were discussed. While our final results are calculated for abrupt interfaces, we showed that a smooth interface does not affect our findings.

$$
* * *
$$

HMA and HB acknowledge the support of the Saudi Center for Theoretical Physics (SCTP) for their generous support and the support of King Fahd University of Petroleum and Minerals under physics research group projects RG1306-1 and RG01306-2. This work is supported by the Flemish Science Foundation (FWO-Vl) by a $\mathrm{PhD}$ grant (BVD) and a post-doctoral fellowship (MZ).

\section{REFERENCES}

[1] Rozhkov A. V., Giavaras G., Bliokha Y. P., Freilikher V. and Nori F., Phys. Rep., 503 (2011) 77.

[2] Castro Neto A. H., Guinea F., Peres N. M. R., Novoselov K. S. and Geim A. K., Rev. Mod. Phys., 81 (2009) 109.

[3] Cranford S., Sen D. and Buehler M. J., App. Phys. Lett., 95 (2009) 123121.

[4] Costa A. T., Ferreira M. S., Hallam Toby, Duesberg Georg S. and Castro Neto A. H., EPL, 104 (2013) 47001.

[5] Hallam T., Shakouri A., Poliani E., Rooney A. P., Ivanov I., Potie A., TAYlor H. K., Bonn M., Turchinovich D., Haigh S. J., Maultzsch J. and Duesberg G. S., Nano Lett., 15 (2015) 857.

[6] Wu Y. H., Tseng P., Hsieh P., Chou H. and Tai N., ACS Appl. Mater. Interfaces, 7 (2015) 9453.

[7] da Costa D. R., Chaves A., Sena S. H. R., Farias G. A. and Peeters F. M., Phys. Rev. B, 92 (2015) 045417.

[8] Zarenia M., Chaves A., Farias G. A. and Peeters F. M., Phys. Rev. B, 84 (2011) 245403.

[9] González J. W., Santos H., Pacheco M., Chico L. and Brey L., Phys. Rev. B, 81 (2010) 195406.

[10] Bliokh Y. P., Freilikher V. and Nori F., Phys. Rev. $B, 81$ (2010) 075410 .

[11] McCann E. and Fal'ko V. I., Phys. Rev. Lett., 96 (2006) 086805 .

[12] For a recent review on graphene bilayer systems see: Rozhkov A. V., Sboychakov A. O., Rakhmanov A. L. and Nori F., arXiv:1511.06706.
[13] Ohta T., Bostwick A., Seyller T., Horn K. and Rotenberg E., Science, 313 (2006) 951.

[14] Castro E. V., Novoselov K. S., Morozov S. V., Peres N. M. R., Lopes dos Santos J. M. B., Nilsson J., Guinea F., Geim A. K. and Castro Neto A. H., J. Phys.: Condens. Matter, 22 (2010) 175503.

[15] Zhang Y., Tang T.-Ta., Girit C., Hao Z., Martin M. C., Zettl A., Crommie M. F., Shen Y. Ron and WANG F., Nature, 459 (2009) 820.

[16] Lopes dos Santos J. M. B., Peres N. M. R. and Castro Neto A. H., Phys. Rev. Lett., 99, 256802.

[17] Mele E. J., Phys. Rev. B, 81, 161405(R).

[18] Lopes dos Santos J. M. B., Peres N. M. R. and Castro Neto A. H., Phys. Rev. B, 86, 155449.

[19] Sboychakov A. O., Rakhmanov A. L., Rozhkov A. V. and Nori Franco, Phys. Rev. B, 92, 075402.

[20] Ju L., Shi Z., Nair N., LV Y., Jin C., Velasco J. jr., Ojeda-Aristizabal C., Bechtel H. A., Martin M. C., Zettl A., Analytis J. and Wang F., Nature, 520 (2015) 650.

[21] Yin L., Li S., Qiao J., Zuo W. and He L., arXiv:1509.04405 (2015).

[22] Yan W., Li S., Yin L., Qiao J., Nie J. and He L., arXiv:1502.00785 (2015).

[23] Basov D. N. and Fogler M. M., Rev. Mod. Phys., 86 (2014) 959.

[24] Cooper D. R., D'Anjou B., Ghattamaneni N., Harack B., Hilke M., Horth A., Majlis N., Massicotte M., Vandsburger L., Whiteway E. and Yu V., ISRN Condens. Matter Phys., 2012 (2012) 501686.

[25] Van Duppen B. and Peeters F. M., Phys. Rev. B, 87 (2013) 205427.

[26] Snyman I. and Beenakker C. W. J., Phys. Rev. B, 75 (2007) 045322.

[27] Partoens B. and Peeters F. M., Phys. Rev. B, 74 (2006) 075404.

[28] Lobato I. and Partoens B., Phys. Rev. B, 83 (2011) 165429.

[29] XU Y., Li X. and Dong J., Nanotechnology, 21 (2010) 065711.

[30] Tabert C. J. and Nicol E. J., Phys. Rev. B, 86 (2012) 075439 .

[31] Barbier M., Vasilopoulos P. and Peeters F. M., Phys. Rev. B, 82 (2010) 235408.

[32] Van Duppen B., Sena S. H. R. and Peeters F. M., Phys. Rev. B, 87 (2013) 195439.

[33] Grover S., Ghosh S. and Sharma M., Model. Simul. Mater. Sci. Eng., 20 (2012) 045010; GHazanfari S. R., Acta Phys. Pol. A, 123 (2013) 148; BAhlouli H., Choubabi E. B. and Jellal A., EPL, 95 (2011) 17009.

[34] Kleptsyn V., Okunev A., Schurov I., Zubov D. and Katsnelson M. I., Phys. Rev. B, 92 (2015) 165407.

[35] Blanter Y. M. and Büttiker M., Phys. Rep., 336 (2000) 1. 